\title{
Análise espacial dos fatores da equação universal de perda de solo em área de nascentes
}

\author{
Daniela Popim Miqueloni(1), Célia Regina Paes Bueno(2) e Antonio Sergio Ferraudo(3)
}

\begin{abstract}
(1)Universidade Estadual Paulista Júlio de Mesquita Filho (Unesp), Faculdade de Ciências Agrárias e Veterinárias (FCAV), Departamento de Solos e Adubos, Via de Acesso Prof. Paulo Donato Castellane, s/no, CEP 14884-900 Jaboticabal, SP. E-mail: danimique@yahoo.com.br, crbueno@fcav.unesp.br ${ }^{(3)}$ Unesp, FCAV, Departamento de Ciências Exatas. E-mail: ferraudo@fcav.unesp.br
\end{abstract}

Resumo - O objetivo deste trabalho foi avaliar a perda de solo de área de nascentes da Microbacia do Córrego do Tijuco, SP. Foi utilizada a análise espacial dos fatores da equação universal da perda de solo (EUPS), em integração com análise de componentes principais e geoestatística. A perda de solo média, estimada para a área, foi de $118,5 \mathrm{Mg} \mathrm{ha}^{-1}$ por ano, considerada alta. Próximo à zona urbana, houve alta interação dos fatores erosividade da chuva e práticas conservacionistas, o que evidencia grande perda de solo, em razão da concentração da água proveniente da camada impermeabilizada urbana, com alta velocidade de escoamento. Nos divisores de águas, a atuação da erodibilidade foi proeminente, em contraste com o fator topográfico. Foram observadas áreas com atuação conjunta destes fatores, inclusive em locais de inclinação suave, porém com alto potencial natural de erosão. A interação das análises multivariadas e geoestatística permite a estratificação da área, identifica locais com propriedades específicas quanto à perda de solo, e espacializa os fatores do processo erosivo e suas interações ao longo do relevo.

Termos para indexação: análise de componentes principais, geoestatística, processo erosivo.

\section{Spatial analysis of universal soil loss equation factors of a watershed area relief}

\begin{abstract}
The objective of this work was to evaluate the soil loss of an area of springs in the Microbacia do Córrego do Tijuco, SP, Brazil. Spatial analysis of the universal soil loss equation (USLE) factors, in integrated with principal component analysis and geostatistics, was used. The average soil loss estimated for the area was $118.5 \mathrm{Mg} \mathrm{ha}^{-1}$ per year, which is considered high. Near the urban zone, there was a high interaction of rainfall erosivity and conservation practices, which shows a high-soil loss, due to the concentration of water from impervious urban layer with a high-flow velocity. In the water partings, the performance of erodibility was prominent, in contrast to the topographic factor. Areas were observed with joint action of these factors, including sites with gentle slope, but with a high, natural potential of erosion. The interaction of multivariate and geostatistic analyses allows the area stratification, identifies locations with specific properties as to soil loss, and spatialises the erosion factors and their interactions along the landscape.
\end{abstract}

Index terms: principal component analysis, geostatistics, erosive process.

\section{Introdução}

A ocupação urbana e o manejo agropecuário inadequados têm contribuído para deflagrar a exaustão dos recursos naturais (Bertoni \& Lombardi Neto, 2008). A preocupação com o planejamento do uso do solo e a manutenção da qualidade da água e dos remanescentes florestais é um fato a ser enfrentado pela sociedade. Tendo-se em vista o grande impacto causado pela agricultura na qualidade e quantidade destes recursos, ações de mitigação da perda de solo e conservação de recursos são necessários e, para isso, a estimativa dos processos erosivos é uma ferramenta valiosa.

Com o intuito de contribuir para essas estimativas, alguns estudos têm mostrado que os processos superficiais de perda de solo, transporte e deposição de materiais, processos pedogenéticos e climáticos atuam na variabilidade das propriedades dos solos, condicionados em grande parte pelo relevo (Sanchez et al., 2005, 2009; Souza et al., 2005). Assim, o comportamento espacial das características dos solos está diretamente relacionado às pedoformas do relevo, ao controlar o sentido e a intensidade do fluxo superficial de água (Leão et al., 2010). Além disso, a cobertura vegetal e as práticas conservacionistas, como o uso de terraços e plantio em nível, reduzem significativamente as perdas de solo pelo processo erosivo (Albuquerque et al., 2005; Inácio et al., 2007). As características intrínsecas de cada tipo de solo também interferem no processo erosivo, principalmente quando associado

Pesq. agropec. bras., Brasília, v.47, n.9, p.1358-1367, set. 2012 
ao relevo (Bueno \& Stein, 2004), que age de forma expressiva nos demais fatores de perda de solo (Campos et al., 2008; Weill \& Sparovek, 2008).

A equação universal da perda de solo (EUPS) é um modelo amplamente difundido para a estimativa de perda de solo, principalmente por ser prático e utilizar variáveis de fácil obtenção. Diversos estudos consideram a EUPS e seus fatores como uma ferramenta de boa aplicabilidade e estimativa, que fornece parâmetros para o planejamento do uso e ocupação do solo. Mendonça et al. (2006) concluíram, por meio da EUPS, que o uso e ocupação de cerca de $60 \%$ da microbacia estudada foram adequados. Farinasso et al. (2006) determinaram a perda de solo pela EUPS, verificaram sua dependência espacial por geoestatística e observaram que as áreas críticas estavam associadas a maiores declividades, solos rasos e uso e manejo inadequados.

O modelo mostra grande sensibilidade aos fatores topográfico e de erosividade da chuva, principalmente em áreas de relevo mais movimentado (Wischmeier \& Smith, 1978). Outros estudos também salientam a ampliação da participação do relevo na aceleração do processo de perda de solo, em função do aumento do valor do fator topográfico (Silva et al., 2005; Campos et al., 2008; Weill \& Sparovek, 2008).

A análise de componentes principais (ACP), é uma ferramenta de análise exploratória, de grande utilização em diversas áreas (Silva et al., 2010), na qual se determina novas variáveis com base na matriz de correlação dos dados existentes, gerando novas informações de forma condensada de forma a explicar a maior parte da variabilidade dos dados originais. Aplicada aos fatores da perda de solo, a ACP fornece a interação de cada fator com o processo erosivo, mede a influência de cada um sem a interferência da sensibilidade do modelo aplicado em áreas de maior movimentação de relevo, além de simplificar a descrição do conjunto de variáveis interrelacionadas. A geoestatística, neste caso, atua na verificação e quantificação do grau de dependência espacial dos dados gerados por meio da aplicação da ACP.

Considerando-se a ampla variabilidade dos fatores de perda de solo e a grande influência do relevo e da erosividade das chuvas no processo erosivo, a aplicação destas ferramentas ao estudo da perda de solo pode oferecer novos parâmetros para a determinação da interação de cada fator associado ao processo erosivo.
O objetivo deste trabalho foi estimar a perda de solo de uma área de nascentes e analisar a variabilidade espacial dos fatores de perda de solo ao longo do relevo.

\section{Material e Métodos}

A área de estudo compreende a região de nascentes da Microbacia do Córrego do Tijuco e ocupa aproximadamente 1.540 ha, no Município de Monte Alto, SP, pertencente à sub-bacia do Rio Mogi-Guaçu, nas coordenadas centrais $764500 \mathrm{E}$ e $7649000 \mathrm{~S}$ (UTM, Córrego Alegre Sul, 22K). O clima, conforme a classificação de Köppen, é mesotérmico de inverno seco (Cwa), com precipitações anuais que variam entre 1.100 e $1.700 \mathrm{~mm}$ e temperatura média anual de $20^{\circ} \mathrm{C}$. Localizada no Planalto Ocidental, a zona do Planalto de Monte Alto, é constituída por arenitos e arenitos com cimento carbonático, da Formação Adamantina e Marília, respectivamente, pertencentes ao Grupo Bauru, com altitude entre 410 a $740 \mathrm{~m}$. Os solos predominantes são da classe dos Argissolos Vermelho-Amarelos eutróficos, com ou sem caráter abrúptico, textura arenosa ou francoarenosa, relevo suave-ondulado e ondulado, horizonte A moderado. O uso e ocupação se dividem em área urbana e cultivo agrícola com cana-de-açúcar, pastagem, frutas e hortaliças.

Para a determinação da erodibilidade, foram coletadas, em junho de 2009, 239 amostras de solo, georreferenciadas a partir de uma malha regular de $250 \mathrm{~m}$ de distância entre os pontos, da profundidade de 0 a $0,20 \mathrm{~m}$. As amostras foram secas ao ar, destorroadas e passadas em peneira com malha de $2 \mathrm{~mm}$ de abertura. A análise granulométrica foi realizada de acordo com o método da pipeta (Day, 1965), com posterior fracionamento composto da fração areia. A matéria orgânica do solo foi determinada de acordo com o método colorimétrico descrito em Cantarella et al. (2001) (Tabela 1).

Tabela 1. Estatística descritiva dos teores (\%) de argila, silte, matéria orgânica (MO), areia grossa (AG), areia média $(\mathrm{AM})$, areia fina (AF), areia muito fina (AMF).

\begin{tabular}{lccccccc}
\hline Variáveis & Argila & Silte & MO & AG & AM & AF & AMF \\
\hline Média & 13,44 & 5,82 & 0,90 & 1,96 & 18,43 & 41,89 & 18,54 \\
DP & 5,41 & 3,02 & 0,49 & 1,31 & 5,52 & 5,76 & 4,64 \\
CV & 40,21 & 51,86 & 54,84 & 66,53 & 29,94 & 13,74 & 25,02 \\
\hline
\end{tabular}

Número de amostras para as variáveis: N, 239; DP, desvio-padrão; CV, coeficiente de variação. 
A perda de solo média anual (A) foi estimada conforme Wischmeier \& Smith (1978), pela equação universal de perda de solo (EUPS), expressa em $\mathrm{Mg} \mathrm{ha} \mathrm{h}^{-1}$ por ano, conforme $\mathrm{A}=\mathrm{R} \times \mathrm{K} \times \mathrm{LS} \times \mathrm{C} \times \mathrm{P}$, em que: $\mathrm{R}$ é o fator de erosividade da chuva, expresso em MJ mm ha- $\mathrm{h}^{-1}$; $\mathrm{K}$ é o fator erodibilidade do solo, expresso em $\mathrm{Mg} \mathrm{h} \mathrm{MJ}^{-1} \mathrm{~mm}^{-1}$; LS é o fator topográfico, adimensional; $\mathrm{C}$ é o fator de uso e manejo, adimensional; e Pé o fator de práticas conservacionistas, adimensional.

O fator erosividade da chuva (R) foi obtido pelo programa NetErosividade (Moreira et al., 2006), com os valores desenvolvidos para o Estado de São Paulo de acordo com equação proposta por Lombardi Neto \& Moldenhauer (1992), citados por Moreira et al. (2006). O programa leva em consideração a precipitação média mensal e anual de 138 estações pluviométricas distribuídas pelo Estado de São Paulo. Para cada ponto amostral, segundo suas coordenadas geográficas, o programa fornece um valor de $\mathrm{R}$ correspondente, o que varia de acordo com a altitude e interfere diretamente nas características da precipitação (Moreira et al., 2006).

O fator erodibilidade do solo (K), em $\mathrm{Mg} \mathrm{h} \mathrm{MJ}^{-1} \mathrm{~mm}^{-1}$, foi obtido pelo modelo proposto por Denardin (1990) como $\mathrm{K}=7,48 \times 10^{-6} \mathrm{M}+4,48059 \times 10^{-3} \mathrm{VP}$ - 6,31175x $10^{-2} \mathrm{DMP}+1,039567 \times 10^{-2} \mathrm{VR}$, em que: $\mathrm{M}(\%)=($ areia muito fina + silte $) \times($ areia muito fina + silte + areia muito grossa + areia grossa + areia média + areia fina); VPé o valor da permeabilidade (determinado pelos valores: 1 , muito rápida; 2 , rápida; 3 , moderada; 4, lenta; 5, muito lenta; 6, imperfeitamente drenada); DPM é o diâmetro médio ponderado das partículas de solo menores que $2 \mathrm{~mm}$; e VR $(\%)=$ (areia muito grossa + areia grossa + areia média + areia fina $) \times($ teor de matéria orgânica)/100.

O fator topográfico (LS) foi determinado pela associação do comprimento de rampa e declividade e estimado pela equação proposta por Bertoni \& Lombardi Neto (2008): $\mathrm{LS}=0,00984 \mathrm{C}^{0,63} \times \mathrm{D}^{1,18}$, em que: C é o fator comprimento de rampa (m); e D é o grau do declive $(\%)$.

O fator de uso e manejo (C) foi determinado conforme Bertoni \& Lombardi Neto (2008), com atribuição de valores segundo a razão de perda de um solo cultivado em relação ao mesmo solo descoberto.

O fator de práticas conservacionistas $(\mathrm{P})$ foi obtido pela equação determinada por Lagrotti (2000) como
$\mathrm{P}=0,69947-0,08991 \mathrm{D}+0,01184 \mathrm{D}^{2}-0,000335 \mathrm{D}^{3}$, em que D é a declividade (\%).

$\mathrm{O}$ potencial natural de erosão (PNE), em $\mathrm{Mg} \mathrm{ha}^{-1}$ por ano, foi calculado pela equação $\mathrm{PNE}=\mathrm{R} \times \mathrm{K} \times \mathrm{LS}$.

Os fatores R, K e LS dependem das condições naturais de clima, solo e relevo, enquanto os fatores $\mathrm{C}$ e $\mathrm{P}$ relacionam-se a ações antrópicas, por meio da forma de ocupação e uso-manejo das áreas.

Os fatores de perda de solo obtidos (R, K, LS, C e P) foram analisados de acordo com os procedimentos da estatística descritiva, referentes às medidas de tendência central e variabilidade, bem como pela correlação linear de Pearson quanto às estimativas de perda de solo (A e PNE). As variáveis foram classificadas de acordo com o coeficiente de variação (Warrick \& Nielsen, 1980). As análises descritiva e multivariada foram processadas no programa Statistica 7.0.

Em razão da sensibilidade do modelo quanto aos fatores LS e R (Silva et al., 2005), e por se tratar de uma área de relevo movimentado, os fatores determinados foram padronizados para o processamento da ACP, o que resultou em média nula e variância unitária dos fatores. Este procedimento visou garantir a consistência da técnica, uma vez que as medidas de distância são sensíveis a diferenças de escalas ou de unidades das variáveis, o que reduziu o efeito de sensibilidade do modelo a valores extremos.

A ACP é uma técnica de interdependência e que analisa as variáveis simultaneamente, ou seja, não define as variáveis como dependentes ou independentes. Esta análise gera novas variáveis latentes ou autovalores, definidos como combinações lineares das variáveis originais medidas, chamadas componentes principais. Por meio destes componentes, são determinados seus autovetores, que atribuem peso para cada variável e determinam seu padrão de distribuição no plano bidimensional, formado pelos componentes principais, o que evidencia a influência de cada fator e sua interação com os demais fatores nos diversos pontos da área estudada (Silva et al., 2010). Assim, sua aplicação aos fatores da EUPS simplifica a descrição do conjunto das variáveis que são independentes, porém interrelacionadas em função dos processos de perda de solo, gerando informações confiáveis em poucas dimensões e fornecendo sua atuação conjunta ao longo do relevo.

A ACP foi aplicada às variáveis $\mathrm{R}, \mathrm{K}, \mathrm{LS}, \mathrm{C}$ e $\mathrm{P}$, em função das variáveis perda de solo $(\mathrm{A})$ e potencial 
natural de erosão (PNE), definidas como variáveis suplementares. Estas variáveis foram incluídas na análise, pelo comando principal component and classification analysis (PCCA) do programa Statistica 7.0, com o objetivo de avaliar, dentre os fatores de perda de solo propostos pela EUPS, quais estariam contribuindo com maior peso na ACP. Cabe ressaltar que as variáveis suplementares não foram utilizadas para a extração de componentes, no entanto foram mapeadas e projetadas nos gráficos bidimensionais e serviram de ponto de referência ou, ainda, foram confrontadas com as variáveis ativas. Sua função foi facilitar a compreensão das estruturas subjacentes da relação das variáveis ativas com as variáveis preditoras (suplementares), isto é, a correlação dos fatores com a perda de solo.

A determinação dos componentes principais (CP) foi calculada segundo a equação a seguir:

$\mathrm{r}_{\text {varíávelj }}\left(\mathrm{CP}_{\mathrm{h}}\right)=\frac{\mathrm{a}_{\mathrm{jh}} \sqrt{\lambda_{\mathrm{h}}}}{\mathrm{s}_{\mathrm{j}}}$

em que: a $\mathrm{r}_{\text {variávelj }}\left(\mathrm{CP}_{\mathrm{h}}\right)$ é a correlação da variável $\mathrm{j}$ com o h-ésimo componente principal; $\mathrm{s}_{\mathrm{j}}$ é o desvio-padrão da variável $\mathrm{j} ; \mathrm{a}_{\mathrm{jh}}$ é o coeficiente da variável $\mathrm{j}$, no h-ésimo componente principal; e $\lambda_{\mathrm{h}}$ é o autovalor de cada dimensão $h$.

Os autovetores $(\mathrm{CP} 1, \mathrm{CP} 2, \ldots \mathrm{CPh})$ foram construídos a partir dos autovalores da matriz de covariância dos fatores da perda de solo, em ordem decrescente, em que o primeiro componente principal (CP1) foi o componente que reteve a maior variabilidade do conjunto original dos dados, enquanto o último componente reteve a menor. A variância retida em cada componente principal pode ser calculada segundo modelo a seguir (7):

$$
\mathrm{CP}_{\mathrm{h}}=\frac{\lambda_{\mathrm{h}}}{\operatorname{traço~}(\mathrm{C})} \times 100
$$

em que: $\left(\mathrm{CP}_{h}\right)$ é o componente principal $h ; \lambda_{h}$ é o autovalor $\mathrm{h}$; $\mathrm{C}$ é a matriz de covariância; e traço $(\mathrm{C})=$ $\lambda 1+\lambda 2+\ldots+\lambda h$.

O agrupamento dos pontos foi realizado visualmente, com base nos gráficos bidimensionais dos componentes, em função da distribuição no plano em relação aos fatores de perda de solo. A dependência espacial entre as amostras foi avaliada pela geoestatística para cada componente principal, pelos respectivos escores, com o auxílio do programa GS $+7.0 \mathrm{e}$, com base na pressuposição de estacionariedade da hipótese intrínseca (Vieira, 2000), estimada por

$$
\gamma(\mathrm{h})=\frac{1}{2 \mathrm{~N}(\mathrm{~h})} \sum_{\mathrm{i}=1}^{\mathrm{N}(\mathrm{h})}\left[\mathrm{Z}\left(\mathrm{x}_{\mathrm{i}}\right)-\mathrm{Z}\left(\mathrm{x}_{\mathrm{i}}+\mathrm{h}\right)\right]^{2}
$$

em que: N(h) é o número de pares de observações Z(xi) e $\mathrm{Z}(\mathrm{xi}+\mathrm{h})$, separados por uma distância $\mathrm{h}$.

O gráfico de $\gamma(\mathrm{h})$ pela distância $\mathrm{h}$ foi denominado semivariograma e serviu de base para a determinação dos mapas de isolinhas, delineados no programa Surfer 8.0, bem como das áreas das classes de cada fator, com o dado Córrego Alegre Sul, em UTM fuso 22K.

Aseleção e o ajuste dos modelos dos semivariogramas foram realizados com base na menor soma de quadrados do resíduo, maior coeficiente de determinação e melhor desempenho da validação cruzada (Mello et al., 2005, 2008). O grau de dependência espacial foi determinado pela razão entre a variância $(C)$ e o patamar $\left(\mathrm{C}_{0}+\mathrm{C}\right)$, classificado segundo Cambardella et al. (1994) como: menor que $25 \%$ (fraco); entre 25 e $75 \%$ (moderado); e maior que $75 \%$ (forte).

\section{Resultados e Discussão}

O teste de normalidade de Kolmogov-Smirnov, a $5 \%$ de probabilidade, indicou comportamento normal apenas para o fator $\mathrm{R}$, enquanto para os fatores $\mathrm{C}$ e $\mathrm{P}$ houve tendência à normalidade dos dados, em razão da proximidade das medidas de tendência central (Tabela 2). Os fatores K, LS, PNE e A apresentaram forte assimetria positiva. Mello et al. (2006) também obtiveram dados de PNE e A com distribuição

Tabela 2. Estatística descritiva dos fatores erosividade das chuvas $(\mathrm{R})$, erodibilidade do solo $(\mathrm{K})$, cobertura do solo (C), práticas conservacionistas (P), fator topográfico (LS), potencial natural de erosão (PNE) e perda de solo (A).

\begin{tabular}{lcccccccc}
\hline Variável & Média & Mediana & DP & Variância & CV & AS & Curtose & KS \\
\hline Fator $\mathrm{R}^{(1)}$ & $7.924,30$ & $7.928,30$ & 40,60 & $1.646,30$ & 0,51 & $-0,14$ & $-0,60$ & $0,069^{*}$ \\
Fator $\mathrm{K}^{(2)}$ & 0,04 & 0,02 & 0,05 & 0,003 & 146,06 & 6,01 & 44,53 & 0,230 \\
Fator C $^{(3)}$ & 0,24 & 0,32 & 0,14 & 0,02 & 59,16 & $-1,10$ & $-0,79$ & 0,142 \\
Fator $\mathrm{P}^{(3)}$ & 0,53 & 0,50 & 0,21 & 0,04 & 39,11 & 1,06 & 1,38 & 0,056 \\
Fator LS $^{(3)}$ & 4,03 & 3,35 & 2,90 & 8,39 & 71,80 & 3,12 & 15,08 & 0,148 \\
\hline $\mathrm{PNE}^{(4)}$ & $1.130,70$ & 577,20 & $1.720,60$ & 2.960 .328 & 152,17 & 5,29 & 42,49 & 0,241 \\
$\mathrm{~A}^{(4)}$ & 118,50 & 61,30 & 243,30 & 54.896 & 197,72 & 7,78 & 86,73 & 0,289 \\
\hline
\end{tabular}

Número de amostras para todas as variáveis: N, 239; DP, desvio-padrão; $\mathrm{CV}$, coeficiente de variação; AS, coeficiente de assimetria; KS, Kolmogorov-Smirnov. *Significativo a $5 \%$. ${ }^{(1)} \mathrm{MJ} \mathrm{mm} \mathrm{ha}{ }^{-1} \mathrm{~h}^{-1}$ por ano. ${ }^{(2)} \mathrm{Mg} \mathrm{h}$ $\mathrm{MJ}^{-1} \mathrm{~mm}^{-1} .{ }^{(3)}$ Fatores adimensionais. ${ }^{(4)} \mathrm{Mg}$ ha ${ }^{-1}$ por ano. 
distante da normalidade, principalmente em razão da variabilidade da cobertura vegetal e manejo da área.

$\mathrm{O}$ valor médio do fator $\mathrm{K}$ foi considerado alto, acima de $0,03 \mathrm{Mg} \mathrm{h} \mathrm{MJ}^{-1} \mathrm{~mm}^{-1}$ (Bertoni \& Lombardi Neto, 2008); valores médios semelhantes foram encontrados por Bueno \& Stein (2004) e Silva (2008) para Argissolos Vermelho-Amarelos. $\mathrm{O}$ fator $\mathrm{K}$ apresentou maior influência na determinação da perda de solo principalmente em áreas oriundas de solos com horizonte de acúmulo de argila.

Para o fator LS, aproximadamente $45 \%$ da área apresentou valores altos (Bertoni \& Lombardi Neto, 2008), definidos por maiores declividades e menores comprimentos de rampa, o que indicou maior movimentação do relevo, principalmente nas áreas de nascentes. Silva et al. (2005) também observaram a importância deste fator, em área de relevo movimentado, no Litoral Norte do Estado de São Paulo, o que salienta a potencialização do processo erosivo pela topografia nestas áreas.

Valores moderados foram encontrados em $75 \%$ da área para o fator $\mathrm{P}$ (referentes aos plantios em contorno), $\mathrm{e}$ mostraram que as práticas conservacionistas auxiliaram no controle do processo erosivo, não de forma isolada como observado por Albuquerque et al. (2005). Com cultivos anuais houve perda de aproximadamente 14\% nas áreas de maior declive (acima de 20\%).

Foi observada perda máxima para o fator $\mathrm{C}$ em $75 \%$ da área, correspondente ao cultivo de cana-de-açúcar, pastagens e frutíferas, e $14 \%$ com perda moderada. Aproximadamente $11 \%$ da área apresentou perda mínima para ambos os fatores, o que corresponde às áreas de vegetação permanente e sem manejo. Weill \& Sparovek (2008) também observaram perda máxima de cultura e manejo, associada ao cultivo de cana-de-açúcar, o que indica a necessidade de cultivo em áreas menos suscetíveis à perda de solo.

Os valores de PNE e A mostraram a variabilidade da área, principalmente com relação ao relevo. Mello et al. (2006), em Argissolo Vermelho-Amarelo na região de Monte Alto, obtiveram valores menores de PNE, em razão das condições menos movimentadas de relevo. Campos et al. (2008) observaram forte relação espacial entre o PNE e a perda de solo com o fator topográfico, o que indicou a dependência dos fatores de erosão em relação ao relevo.

$\mathrm{O}$ valor médio obtido para o fator $\mathrm{R}$, na área de estudo, foi de $7.924 \mathrm{MJ} \mathrm{mm} \mathrm{h}^{-1} \mathrm{ha}^{-1}$ por ano. Valores semelhantes foram encontrados por Moreira et al. (2006), para o Município de Monte Alto, e por Bertoni \& Lombardi Neto (2008), que determinaram valores entre 7.500 e $7.750 \mathrm{MJ} \mathrm{mm} \mathrm{h}^{-1} \mathrm{ha}^{-1}$ por ano. Segundo Inácio et al. (2007), um dos principais agentes ativos na erosão do solo é a chuva, quando se relaciona a magnitude do processo erosivo principalmente com a cobertura do solo e declividade do terreno.

De acordo com a classificação do coeficiente de variação (CV), proposta por Warrick \& Nielsen (1980), apenas o fator R obteve CV baixo (menor que $12 \%$ ). Os fatores $\mathrm{C}$ e $\mathrm{P}$ apresentaram CV médio (entre 12,1 e $60 \%$ ), e os fatores K e LS, PNE e A apresentaram CV alto (acima de 60\%). Este comportamento assimétrico das variáveis pode ser resultado da variabilidade das condições de cobertura vegetal, manejo e pedoforma da área (Souza et al., 2003, 2005; Mello et al., 2006).

A matriz de correlação de Pearson, com $95 \%$ de confiança entre o PNE e seus fatores, mostrou alta correlação com o fator $\mathrm{K}$, seguido pelo fator $\mathrm{LS}$ (Tabela 3). Para o A, somente o fator K apresentou maior correlação. Campos et al. (2008) observaram, em área de Latossolos, alta correlação do A e PNE com o fator LS, o que corrobora a importância do fator topográfico no processo erosivo.

A análise de componentes principais, aplicada aos fatores de perda de solo, em função das variáveis suplementares A e PNE, discriminou as amostras de solo e indicou que três autovalores foram suficientes para condensar $74,94 \%$ variabilidade contida nos dados originais - 35,22\% no primeiro componente principal, $20,49 \%$ no segundo componente e $19,23 \%$ no terceiro componente - (Tabela 4). A contribuição da ACP, neste caso, foi a ordenação das variáveis, segundo sua importância no processo erosivo ao longo do relevo, o que simplificou os resultados em poucas dimensões (três componentes principais) em relação ao número de fatores de perda de solo.

No primeiro componente principal (CP1), a discriminação relevante ou de maior carga foi a da interação dos fatores $\mathrm{R}, \mathrm{Pe} \mathrm{C}$, em que os dois primeiros

Tabela 3. Coeficiente de correlação do potencial natural de erosão (PNE) e da equação universal da perda de solo (A) e seus fatores.

\begin{tabular}{lccccc}
\hline Variável & Fator R & Fator K & Fator LS & Fator C & Fator P \\
\hline A & $-0,086$ & 0,740 & 0,083 & 0,298 & $-0,074$ \\
PNE & 0,066 & 0,731 & 0,451 & - & - \\
\hline
\end{tabular}


se correlacionam diretamente ao PNE, e o último diretamente com o A (valores negativos de cargas do A e fator $\mathrm{C}$ e valores positivos do PNE e dos fatores $\mathrm{R} \mathrm{e}$ $\mathrm{P})$. Tal comportamento dos fatores antrópicos mostrou que quanto menor a exposição do solo, em relação à erosividade da chuva, menor o efeito do uso e manejo. Isto é, a perda de solo na área foi fortemente influenciada pela chuva e pelo manejo e foi minimizada pela atuação da cobertura vegetal. Assim, também, no estudo de Weill \& Sparovek (2008), os fatores antrópicos foram responsáveis pela ordem de grandeza da estimativa da perda de solo, quando mantido constante o fator R.

Para o segundo componente principal (CP2), em que esteve contido o maior poder discriminante dos fatores naturais, observou-se a correlação direta do fator $\mathrm{K}$ com a perda de solo, o fator LS teve comportamento inverso. Resultados semelhantes foram observados por Salchow \& Lal (2001), que indicaram os fatores naturais como os de maior influência nos componentes principais quanto à produtividade das culturas.

No terceiro componente principal (CP3), em que está contida a discriminação relevante do potencial natural de erosão e os fatores K e LS, a correlação ocorre de forma direta, o que mostra a atuação conjunta dos fatores K e LS na perda potencial de solo em determinadas áreas. Campos et al. (2008), da mesma forma, observaram a maior dependência dos fatores naturais de erosão ao relevo em área de Latossolos. Silva (2008), independentemente do tipo de solo, observou maior influência do fator topográfico e menor

Tabela 4. Autovalores e autovetores da matriz de correlação dos componentes principais $(\mathrm{CP})$ e dos fatores erosividade das chuvas (R), erodibilidade do solo (K), cobertura do solo $(C)$, práticas conservacionistas $(\mathrm{P})$, fator topográfico (LS), potencial natural de erosão (PNE) e perda de solo (A).

\begin{tabular}{lccccc}
\hline Comp. da variância & CP1 & CP2 & CP3 & CP4 & CP5 \\
\hline Autovalores & 1,761 & 1,024 & 0,962 & 0,666 & 0,587 \\
Total da variância(\%) & 35,220 & 20,489 & 19,234 & 13,316 & 11,741 \\
Autovalores cumulativos & 1,761 & 2,785 & 3,747 & 4,413 & 5,000 \\
Variância acumulada(\%) & 35,220 & 55,709 & 74,943 & 88,259 & 100,00 \\
\hline Fator R & 0,761 & $-0,158$ & 0,195 & - & - \\
Fator K & $-0,045$ & $-0,791$ & $-0,608$ & - & - \\
Fator C & $-0,717$ & $-0,153$ & 0,174 & - & - \\
Fator P & 0,661 & $-0,371$ & 0,371 & - & - \\
Fator LS & 0,478 & 0,460 & $-0,621$ & - & - \\
\hline PNE $^{(1)}$ & 0,208 & $-0,378$ & $-0,737$ & - & - \\
$\mathrm{A}^{(1)}$ & $-0,182$ & $-0,539$ & $-0,513$ & - & - \\
\hline
\end{tabular}

(1) Variável suplementar; (-) valores de componentes não considerados por sua baixa variância e contribuição dos fatores. interferência da erodibilidade do solo. A projeção das variáveis no gráfico bidimensional, formado pelos autovetores CP1 e CP2 com 55,71\% da variabilidade original dos dados, e de CP1 e CP3 com 54,45\%,

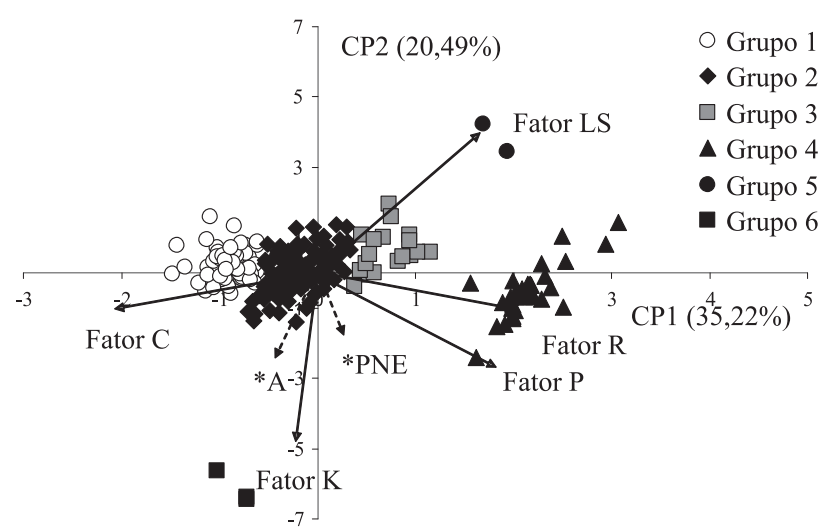

Figura 1. Gráfico biplot para o componente principal 1 (CP1) e o componente principal 2 (CP2) dos fatores de perda de solo projetados no plano. Grupo 1: pontos de amostragem de solo com maior influência do fator C. Grupo 2: pontos de comportamento mediano em relação aos fatores de perda de solo (área central do gráfico). Grupo 3: pontos com tendência à perda de solo pelo fator LS. Grupo 4: pontos com maior influência dos fatores R e P. Grupo 5: pontos com grande influência do fator LS. Grupo 6: grande influência do fator K.

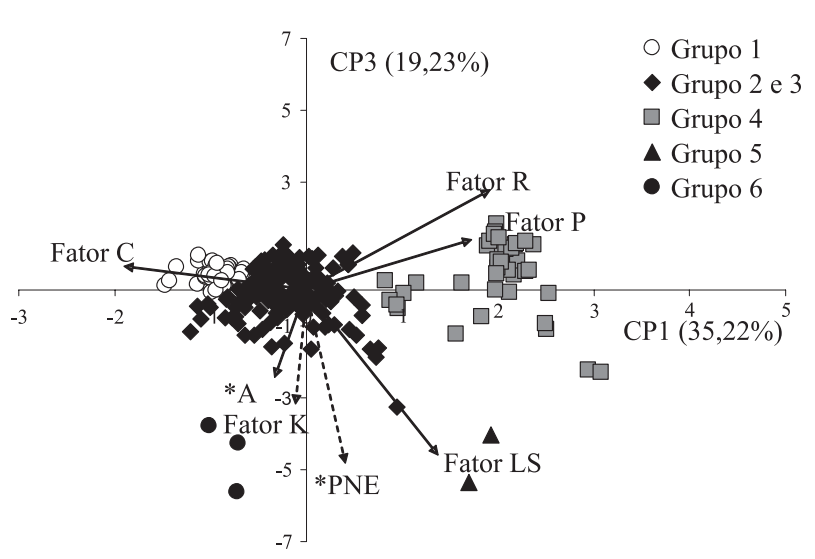

Figura 2. Gráfico biplot para o componente principal 1 (CP1) e o componente principal 3 (CP3) dos fatores de perda de solo projetados no plano. Grupo 1: pontos de amostragem de solo com maior influência do fator C. Grupo 2 e 3: pontos de comportamento mediano em relação aos fatores de perda de solo (área central do gráfico). Grupo 4: pontos com maior influência dos fatores R e P. Grupo 5: pontos com grande influência do fator LS. Grupo 6: grande influência do fator K. 
mostrou a importância dos fatores na discriminação dos grupos das amostras de solos (Figuras 1 e 2).

Nas projeções, foi possível observar a formação de seis grupos, classificados de acordo com as projeções dos componentes principais, o que refletiu seu comportamento com relação aos fatores. Para a primeira projeção (Figura 1), os grupos de maior discriminação foram: Grupo 1, relacionado diretamente ao fator C, e Grupo 4, relacionado aos fatores $\mathrm{R}$ e $\mathrm{P}$, ambos os grupos tiveram comportamento contrastante, isto é, correlação inversa; o Grupo 5 se relacionou diretamente ao fator LS, e o Grupo 6 ao fator K, ambos os grupos se correlacionaram inversamente; os Grupos 2 e 3 mostraram comportamento mediano, com tendência a maiores perdas relacionadas ao fator LS pelo Grupo 3 . Na segunda projeção (Figura 2), o comportamento dos Grupos 1 e 4 foi mantido. Os Grupos 5 e 6 passaram a se relacionar diretamente, o que indica a atuação conjunta destes fatores. Os grupos 2 e 3 mantiveram o comportamento mediano. A atuação e interação destes fatores, no plano bidimensional, refletiram diretamente seu comportamento na área segundo as pedoformas do relevo.

Para espacializar seu comportamento e interações ao longo do relevo, a análise geoestatística foi aplicada aos três primeiros componentes principais, pelos respectivos escores gerados a partir dos fatores de perda de solo (Tabela 5). A dependência espacial foi observada para os três primeiros componentes principais, ou seja, o aumento gradual da semivariância até a distância em que houve estabilização (patamar). O alcance do CP1 indicou dependência espacial da atuação em conjunto dos fatores $\mathrm{R}$ e $\mathrm{P}$ com o contraste visível do fator $\mathrm{C}$ contido neste componente. $\mathrm{O}$ alcance de $\mathrm{CP} 2$ mostrou dependência espacial dentro do âmbito de atuação do contraste entre os fatores K e LS. E o alcance para CP3 mostrou atuação direta dos fatores K e LS com a perda de solo em determinadas áreas. A proximidade dos alcances do $\mathrm{CP} 2$ e $\mathrm{CP} 3$ pode ser explicada pela

Tabela 5. Modelos e parâmetros dos semivariogramas ajustados para cada componente principal.

\begin{tabular}{lccccccc}
\hline Variáveis & Modelo & $\mathrm{C}_{0}$ & $\mathrm{C}_{0}+\mathrm{C}$ & $\mathrm{a}$ & $\mathrm{C} /\left(\mathrm{C}_{0}+\mathrm{C}\right)$ & $\mathrm{R}^{2}$ & $\mathrm{SQR}$ \\
\hline $\mathrm{CP} 1$ & Gaussiano & 0,2260 & 0,914 & 1060,02 & 75,3 & 0,993 & 0,002 \\
$\mathrm{CP} 2$ & Exponencial & 0,2905 & 0,821 & 592,56 & 64,6 & 0,536 & 0,053 \\
$\mathrm{CP} 3$ & Exponencial & 0,1108 & 0,494 & 642,00 & 77,6 & 0,813 & 0,011 \\
\hline
\end{tabular}

$\mathrm{C}_{0}$, efeito pepita; $\mathrm{C}_{0}+\mathrm{C}$, patamar; a, alcance; $\mathrm{C} /\left(\mathrm{C}_{0}+\mathrm{C}\right)$, índice de dependência espacial (IDE); $\mathrm{R}^{2}$, coeficiente de determinação; $\mathrm{SQR}$, soma dos quadrados dos resíduos. influência dos fatores naturais como discriminantes em ambos. Os pontos localizados dentro destas distâncias são semelhantes e, quanto menor a distância, maior a dependência entre os pontos (Vieira, 2000).

O índice de dependência espacial, de acordo com Cambardella et al. (1994), mostrou forte dependência espacial para CP1 e CP3 (acima de 75\%). Para CP2, foi moderada (64\%), o que pode ser atribuído à localização pontual da atuação dos fatores de maior carga neste componente.

Os mapas de isolinhas gerados refletiram 74,94\% da variabilidade dos fatores de perda de solo contidos na área (Figura 3). A distribuição dos componentes principais revelou maior influência da erodibilidade e do relevo sobre a perda de solo e mostrou a atuação dos fatores K e LS na maior parte da área contida em dois componentes principais. Solos arenosos possuem maior permeabilidade, contudo, a presença de horizonte com gradiente textural restringe a infiltração da água, e a baixa coesão de partículas facilita o desprendimento e transporte, o que pode resultar em aumentos significativos de erosão (Toy et al., 2002). O relevo também se mostra atuante, uma vez que compõe a paisagem em diferentes formas, o que resulta no direcionamento de fluxo do escoamento superficial (Salchow \& Lal, 2001). A resistência ao processo de erosão também está relacionada à fase de transporte dos sedimentos, diretamente ligados à velocidade da água, cobertura do solo e relevo, que controla o sentido dos fluxos de água superficial e subsuperficial (Leão et al., 2010; Oliveira et al., 2012).

A distribuição dos fatores $\mathrm{R}$ e $\mathrm{P}$, em contraste com C contida em CP1, mostra menor variabilidade espacial, com maior alcance, e aparece altamente concentrada próximo à área urbana, nas maiores cotas do terreno (Figura 3 A). Tal efeito pode ser explicado pela impermeabilização da área de entorno, que impede a infiltração da água da chuva e potencializa seu escoamento superficial. Os pontos de concentraçãodesta água oriunda da superfície impermeabilizada tornam-se locais sensíveis, onde a perda de solo é pronunciada, em consequência da alta velocidade de escoamento da água (Toy et al., 2002). O contraste dos fatores K e LS, contido em $\mathrm{CP} 2$, teve maior variabilidade espacial em relação a $\mathrm{CP} 1$ e mostrou a atuação do fator $\mathrm{K}$ em áreas específicas (Figura $3 \mathrm{~B}$ ). Estes fatores apareceram em escala gradual com o fator topográfico, em áreas de menor altitude, principalmente em zonas côncavas, 
onde a influência do fator $\mathrm{K}$ foi menor. Estas áreas, com grande deposição de material sedimentar, mostraram o papel do relevo no deslocamento de sedimentos, o que evidencia a variação de perda de solo em relação

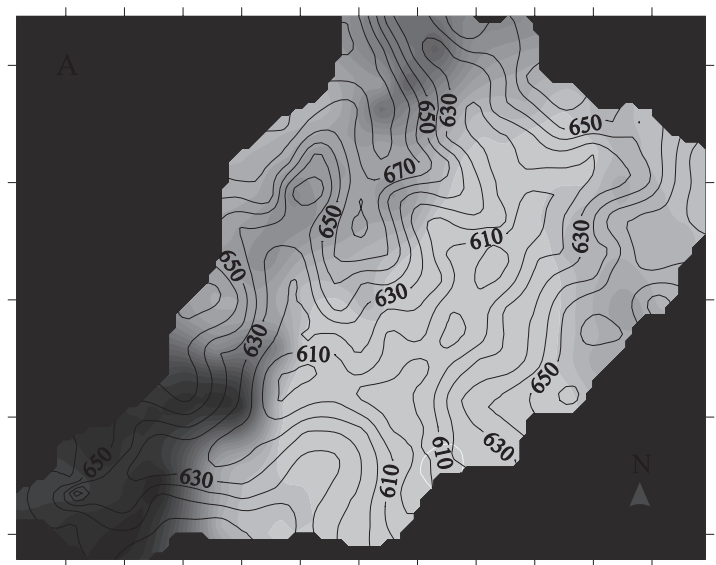

Fatores

R e P

3,0

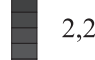

2,2

1,4

0,6

$-0,2$

$-1,0$

$-1,8$

Fator C
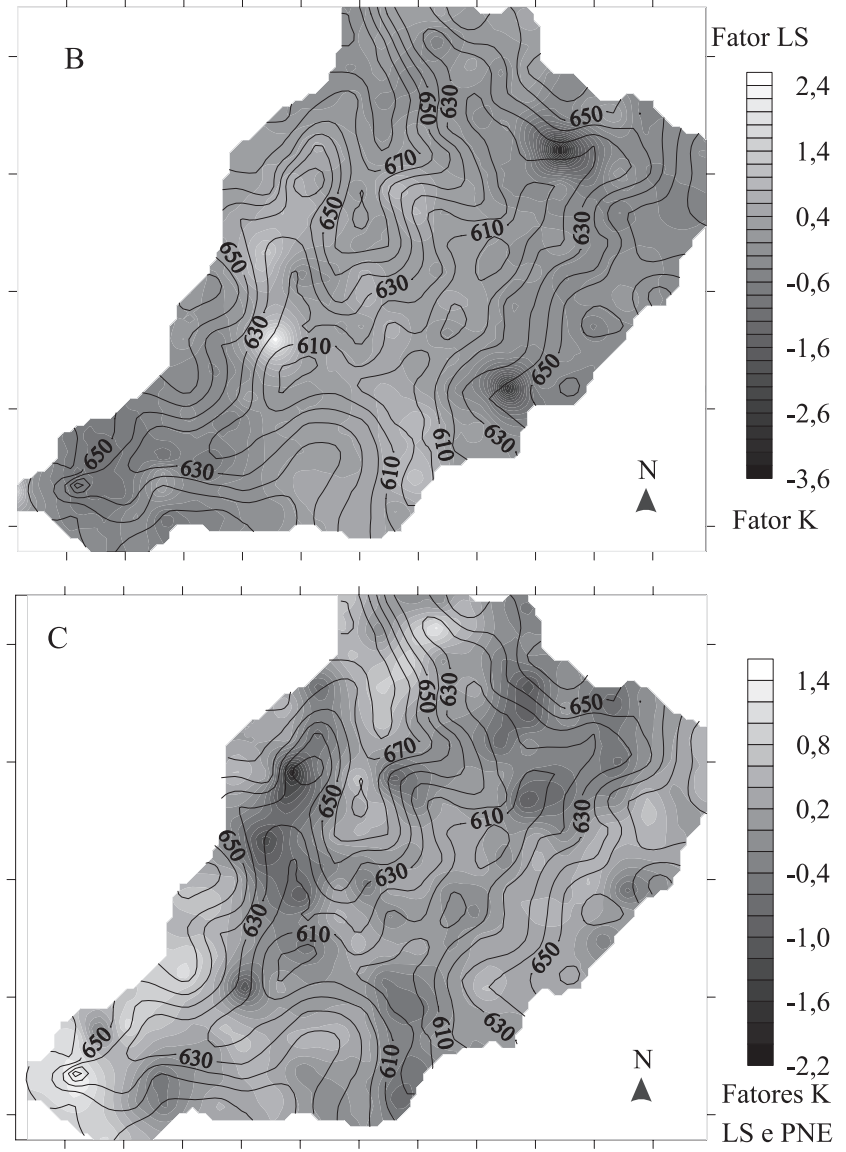

Figura 3. A, distribuição da atuação dos fatores contida em CP1 [(ם) fatores R e P e ( $\square$ ) fator C]; B, distribuição da atuação dos fatores contida em CP2 [( $\square$ ) fator LS e ( $\square$ ) fator $\mathrm{K}$ ]; $\mathrm{C}$, distribuição da atuação dos fatores contidos em CP3 [(ם) fatores K, LS e PNE], em mapa planialtimétrico e suas cotas em curvas a cada $20 \mathrm{~m}$. à paisagem. Este resultado se aproxima do encontrado por Salchow \& Lal (2001), que observaram correlação entre o fator de erodibilidade e o fator topográfico, ambos em atuação direta na produtividade de culturas. Souza et al. (2003) e Sanchez et al. (2009) também observaram maior variabilidade espacial em áreas côncavas, em consequência do acúmulo de materiais. Em áreas pontuais, com forte característica convexa, isto é, nos divisores de águas, a atuação do fator $\mathrm{K}$ foi dominante, o que revelou menor influência do fator topográfico.

A distribuição dos fatores $\mathrm{K}$ e $\mathrm{LS}$, diretamente relacionados ao PNE contido em CP3, também possuiu maior variabilidade espacial e encontrou-se em áreas pontuais, principalmente nas encostas de declive mais suavizado e côncavas (Figura 3 C). A erosividade da chuva atua diretamente nestas pedoformas, uma vez que, em tais setores da paisagem, a energia do escoamento superficial é potencializada, além de o fator topográfico acentuar sua atuação em áreas arenosas (Toy et al., 2002). Nestas áreas, de concavidade em encostas suaves, a atuação contrastante dos grupos com maior especificidade foi destacada em função do potencial natural de erosão (PNE).

Assim, a área se mostrou frágil quanto ao processo erosivo e, de acordo com a correlação de Pearson (Tabela 3), isso ocorreu em função do fator $\mathrm{K}$, em consequência do caráter textural arenoso/médio de baixa coesão do solo. A análise de componentes principais, em razão da padronização dos dados, que reduziu a sensibilidade da EUPS aos fatores topográficos e de erosividade da chuva, indicou a atuação do fator $\mathrm{K}$ em conjunto com o fator topográfico em pontos específicos, que abrangiam pedoformas côncavas, declividades suaves e divisores de águas. Foi observada, ainda, a influência dos demais fatores de perda de solo em zonas específicas da paisagem, como os fatores $\mathrm{R}$ e $\mathrm{P}$ em contraste com $\mathrm{C}$, em área de concentração de água proveniente da zona urbana. Isto permitiu a delimitação de zonas de atuação e manejo de acordo com as pedoformas do relevo e, consequentemente, de seu comportamento erosivo (Sanchez et al., 2005, 2009).

\section{Conclusões}

1. A perda de solo estimada para a área estudada da Microbacia do Córrego do Tijuco é considerada alta. 
2. A análise de componentes principais, em conjunto com a geoestatística, permite a avaliação espacial da perda de solo e seus fatores e contribui para a análise integrada da atuação destes fatores ao longo do relevo da área, bem como do uso da terra.

3. Os fatores da perda de solo se destacam pela atuação localizada, com influência dos fatores $\mathrm{K}$ (erodibilidade) e LS (topográfico) nas áreas cultivadas e cobertas por vegetação, principalmente em pedoformas específicas do relevo, e pela concentração dos fatores $\mathrm{R}$ (erosividade), $\mathrm{P}$ (práticas conservacionistas) e C (uso e manejo) próximos a zonas urbanas.

\section{Referências}

ALBUQUERQUE, A.W. de; M. FILHO, G.; SANTOS, J.R.; COSTA, J.P.V.; SOUZA, J.L. Determinação de fatores da equação universal de perda de solo em Sumé, PB. Revista Brasileira de Engenharia Agrícola e Ambiental, v.9, p.153-160, 2005.

BERTONI, J.; LOMBARDI NETO, F. Conservação do solo. 6.ed. São Paulo: Ícone, 2008. 355p.

BUENO, C.R.P.; STEIN, D.P. Potencial natural e antrópico de erosão na região de Brotas, Estado de São Paulo. Acta Scientiarum. Agronomy, v.26, p.1-5, 2004.

CAMBARDELLA, C.A.; MOORMAN, T.B.; PARKIN, T.B.; KARLEN, D.L.; NOVAK, J.M.; TURCO, R.F.; KONOPKA, A.E. Field-scale variability of soil properties in central Iowa soils. Soil Science Society of America Journal, v.58, p.1501-1511, 1994.

CAMPOS, M.C.C.; MARQUES JUNIOR, J.; MARTINS FILHO, M.V.; PEREIRA, G.T.; SOUZA, Z.M. de; BARBIERI, D.M. Variação espacial da perda de solo por erosão em diferentes superfícies geomórficas. Ciência Rural, v.38, p.2485-2492, 2008.

CANTARELLA, H.; QUAGGIO, J.A.; RAIJ, B. van. Determinação da matéria orgânica. In: RAIJ, B. van; ANDRADE, J.C. de; CANTARELlA, H.; QUAGGIO, J.A. (Ed.). Análise química para avaliação da fertilidade de solos tropicais. Campinas: Instituto Agronômico, 2001. p.173-180.

DAY, P.R . Particle fractionation and particle-size analysis. In: BLACK, C.A. (Ed.). Methods of soil analysis: chemical and microbiological properties. Madison: American Society of Agronomy, 1965. p.545-566. (ASA. Agronomy, 9).

DENARDIN, J.E. Erodibilidade do solo estimada por meio de parâmetros físicos e químicos. 1990. 114p. Tese (Doutorado) Escola Superior de Agricultura Luiz de Queiroz, Piracicaba.

FARINASSO, M.; CARVALHO JUNIOR, O.A. de; GUIMARÃES, R.F.; GOMES, R.A.T.; RAMOS, V.M. Avaliação qualitativa do potencial de erosão laminar em grandes áreas por meio da EUPS - equação universal da perda de solos utilizando novas metodologias em SIG para os cálculos dos seus fatores na região do Alto Parnaíba-PI-MA. Revista Brasileira de Geomorfologia, n.2, p.73-85, 2006.
INÁCIO, E. dos B.; CANTALICE, J.R.B.; NACIF, P.G.S.; ARAUJO, Q.R. de; BARRETO, A.C. Quantificação da erosão em pastagem com diferentes declives na microbacia do Ribeirão Salomea. Revista Brasileira de Engenharia Agrícola e Ambiental, v.11, p.355-360, 2007.

LAGROTTI, C.A.A. Planejamento agroambiental do município de Santo Antônio do Jardim - SP: estudo de caso na microbacia hidrográfica do córrego do Jardim. 2000. 115p. Tese (Doutorado) Universidade de Campinas, Campinas.

LEÃO, M.G.A.; MARQUES JÚNIOR, J.; SOUZA, Z.M. de; PEREIRA, G.T. Variabilidade espacial da textura de um latossolo sob cultivo de citros. Ciência e Agrotecnologia, v.34, p.121-131, 2010.

LOMBARDI NETO, F.; MOLDENHAUER, W.C. Erosividade da chuva: sua distribuição e relação com as perdas de solo em Campinas. Bragantia, v.51, p.189-196, 1992.

MELLO, C.R. de; VIOLA, M.R.; MELLO, J.M. de; SILVA, A.M. da. Continuidade espacial de chuvas intensas no Estado de Minas Gerais. Ciência e Agrotecnologia, v.32, p.532-539, 2008.

MELLO, G. de; BUENO, C.R.P.; PEREIRA, G.T. Variabilidade espacial de perdas de solo, do potencial natural e risco de erosão em áreas intensamente cultivadas. Revista Brasileira de Engenharia Agrícola e Ambiental, v.10, p.315-322, 2006.

MELLO, J.M. de; BATISTIA, J.L.F.; RIBEIRO JUNIOR, P.J.; OLIVEIRA, M.S. de. Ajuste e seleção de modelos espaciais de semivariograma visando à estimativa volumétrica de Eucaliptus grandis. Scientia Forestalis, n.69, p.25-37, 2005.

MENDONÇA, I.F.C.; LOMBADRI NETO, F.; VIÉGAS, R.A. Classificação da capacidade de uso das terras da Microbacia do Riacho Uma, Sapé, PB. Revista Brasileira de Engenharia Agrícola e Ambiental, v.10, p.888-895, 2006.

MOREIRA, M.C.; CECÍLIO, R.A.; PINTO, F. de A. de C.; PRUSKI, F.F. Desenvolvimento e análise de uma rede neural artificial para estimativa da erosividade da chuva para o Estado de São Paulo. Revista Brasileira de Ciência do Solo, v.30, p.1069-1076, 2006.

OLIVEIRA, F.P. de; BUARQUE, D.C.; VIERO, A.C.; MERTEN, G.H.; CASSOL, E.A.; MINELLA, J.P.G. Fatores relacionados à suscetibilidade da erosão em entressulcos sob condições de uso e manejo do solo. Revista Brasileira de Engenharia Agrícola e Ambiental, v.16, p.337-346, 2012.

SALCHOW, E.; LAL, R. Relating crop yields to physiografic attributes in Ohio through principal component analysis. In: STOTT, D.E.; MOTHAR, R.H.; STEINHARDT, G.C. (Ed.). Sustaining the global farm. Washington: International Soil Conservation Organization, 2001. p.272-276.

SANCHEZ, R.B.; MARQUES JÚNIOR, J.; PEREIRA, G.T.; SOUZA, Z.M. de. Variabilidade espacial de propriedades de latossolo e da produção de café em diferentes superfícies geomórficas. Revista Brasileira de Engenharia Agrícola e Ambiental, v.9, p.489-495, 2005.

SANCHEZ, R.B.; MARQUES JUNIOR, J.; SOUZA, Z.M. de; PEREIRA, G.T.; MARTINS FILHO, M.V. Variabilidade espacial de 
atributos do solo e de fatores de erosão em diferentes pedoformas. Bragantia, v.68, p.1095-1103, 2009.

SILVA, A.M. da. Potencial natural de erosão no Município de Sorocaba, São Paulo, Brasil. Revista Internacional de Desastres Naturales, Accidentes e Infraestructura Civil, v.8, p.5-14, 2008.

SILVA, A.M. da; RANZINI, M.; GUANDIQUE, M.E.G.; ARCOVA, F.C.S.; DE CICCO, V. Estudo integrado do processo erosivo numa microbacia experimental localizada no município de Cunha-SP. Geociências, v.24, p.43-53, 2005.

SILVA, S. de A.; LIMA, J.S. de S.; XAVIER, A.C.; TEIXEIRA, M.M. Variabilidade espacial de atributos químicos de um Latossolo Vermelho-Amarelo húmico cultivado com café. Revista Brasileira de Ciência do Solo, v.34, p.15-22, 2010.

SOUZA, C.K.; MARQUES JUNIOR, J.; MARTINS FILHO, M.V.; PEREIRA, G.T. Influência do relevo na variabilidade espacial de um latossolo em Jaboticabal (SP). Revista Brasileira de Ciência do Solo, v.27, p.1067-1074, 2003.

SOUZA, Z.M. de; MARTINS FILHO, M.V.; MARQUES JUNIOR, J.M.; PEREIRA, G.T. Variabilidade espacial de fatores de erosão em Latossolo Vermelho eutroférrico sob cultivo de cana-de-açúcar. Engenharia Agrícola, v.25, p.105-114, 2005.

TOY, T.J.; FOSTER, G.R.; RENARD, K.G. Soil erosion: processes, prediction, measurement, and control. New York: Willey, 2002. $338 \mathrm{p}$.

VIEIRA, S.R. Geoestatística em estudos de variabilidade espacial do solo. In: NOVAIS, R.F. de; ALVAREZ V., V.H.; SCHAEFER, C.E.G.R. (Ed.). Tópicos em ciência do solo. Viçosa: Sociedade Brasileira de Ciência do Solo, 2000. v.1, p.1-54.

WARRICK, A.W.; NIELSEN, D.R. Spatial variability of physical properties in the field. In: HILLEL, D. (Ed.). Applications of soil physics. New York: Academic Press, 1980. p.319-344.

WEILL, M. de A.M.; SPAROVEK, G. Estudo da erosão na Microbacia do Ceveiro (Piracicaba, SP). I - Estimativa das taxas de perda de solo e estudo de sensibilidade dos fatores do modelo EUPS. Revista Brasileira de Ciência do Solo, v.32, p.801-814, 2008.

WISCHMEIER, W.H.; SMITH, D.D. Predicting rainfall erosion losses: a guide to conservation planning. Washington: USDA, 1978. 58p. (Agriculture handbook, 537).

Recebido em 27 de julho de 2011 e aprovado em 30 de agosto de 2012 